\section{Young adolescents' interest in a mental health casual video game}

\author{
Russell Pine $\mathbb{D}$, Kylie Sutcliffe, Simon McCallum and Theresa Fleming
}

Digital Health

Volume 6: 1-7

(C) The Author(s) 2020

Article reuse guidelines:

sagepub.com/journals-

permissions

DOI: $10.1177 / 2055207620949391$

journals.sagepub.com/home/dhj

@SAGE

\title{
Abstract
}

Background: Mental distress and disorders among adolescents are well documented. Despite the array of treatments available, many mental health issues remain untreated and often undiagnosed. In an attempt to narrow the treatment gap, researchers have adapted existing mental health interventions into digital formats. Despite their efficacy in trial settings, however, real-world uptake of digital mental health interventions is typically low. Casual video games (CVGs) are popular among adolescents and may be a promising tool to reduce stress, anxiety and depression.

Aim: We set out to explore young adolescents' views of CVGs and their opinions of mental health CVG prototypes, to help determine whether this idea warrants further investigation.

Methods: Pen and paper feedback forms following a brief presentation to 13-15-year-old adolescents in seven high schools $(n=207)$ followed by more detailed focus groups $(n=42)$ and workshops $(n=21)$ with interested students.

Findings: Across all three methods, participants reported playing CVGs several times a week or day to help relieve stress, feel more relaxed and relieve boredom. Most were also interested in the idea of a mental health CVG. Participants in focus groups and workshops confirmed that playing CVGs was common among themselves and their peers, and that the idea of a CVG with subtle and brief mental health content such as game-linked 'micro messages' was appealing. Participants recommended that the game should have an engaging interface and subtle mental health skills and information.

Conclusions: Findings from this exploratory study suggest that the concept of a mental health CVG appears to be appealing to adolescents. This novel approach should be tested.

\section{Keywords}

Mental health, casual video game, adolescence, co-design

Submission date: 6 February 2020; Acceptance date: 15 July 2020

\section{Introduction}

Globally, 10-20\% of children and adolescents meet the criteria for a mental health disorder. ${ }^{1}$ Despite developments in evidence-based treatments, nearly two-thirds of adolescents with an identified mental disorder never seek help from a health professional. ${ }^{2}$ This is due to external and internal barriers such as lack of available therapists, costs, convenience, stigma and confidentiality concerns. ${ }^{3,4}$ In part to address these issues, digital therapies have been developed. One of the most commonly tested formats, computerised cognitive behaviour therapy (cCBT), has been shown to have comparable effects to face-to-face therapies. ${ }^{5}$
However engagement with digital mental health tools outside of trial settings has typically been disappointing. ${ }^{5-8}$

Systematic, 'top-down' approaches enable users to receive standardised, evidence-based content, however

School of Health, Victoria University of Wellington, Wellington, New Zealand

Corresponding author:

Russell Pine, Victoria University of Wellington, Wellington 6140, New Zealand.

Email: Russell.pine@vuw.ac.nz 
this brings challenges. Internet user preferences and interfaces change rapidly, therefore tools may be outdated by the time they are released. ${ }^{9}$ Many mental health tools aim to provide a full treatment program, which may be poor match with the way people often dip in and out of apps and programs. ${ }^{10,11}$ In addition, some tools retain unnecessary components of traditional therapy within new technologies, a process described as 'skewmorphism'. ${ }^{11}$ For example, weekly full or half hour sessions may work well for clinical appointments but are not necessarily the best use of internet formats. Deep understanding of target users' needs and behaviours, and high quality co-design processes are likely to be important for improving engagement and satisfaction with digital mental health tools. ${ }^{7,8}$

Earlier research with culturally diverse adolescents in New Zealand (NZ) has demonstrated that teens consider mental distress common and yet consider it normal to only seek help in crisis situations..$^{8,12,13}$ Consistent with international research, NZ adolescents reported that they face barriers to mental health support, including not recognising distress as a health need that they could get help for, feeling too hopeless, uncertain or embarrassed to seek help, and more external barriers such as lack of face-to-face treatment, delays in receiving help, or a poor fit with available therapists. ${ }^{8,12,13}$ Youth centred research demonstrates that adolescents make use of a variety of digital tools to support their mental state, such as posting on social media and watching videos or playing video games.

Video games are played by millions of adolescents and adults around the world for a variety of reasons such as to relieve stress and relax. ${ }^{14,15}$ Video games vary from complex multiplayer games, to simple repetitive casual video games (CVGs). Currently, these CVGs such as Candy Crush or Angry Birds are among the most popular type of video game. ${ }^{14} \mathrm{CVGs}$ can be best defined as "fun, fast to access and simple to learn games which require short amounts of time to play and no prior video game knowledge or skills". ${ }^{16}$ CVGs encompass multiple categories and skills, including casual action (motor skill), casual puzzle (logic), idle games (observation), and casual strategy games (strategy). With the diversity of CVGs, they are a popular tool used among broad groups of individuals.

The popularity and potential therapeutic effects of CVGs were consistent with the literature review conducted by members of our team (RP, TF, KS, and $\mathrm{SM})$. Using these ideas as a basis, we consulted with cross-disciplinary researchers and young adolescents and conceptualised a novel tool that capitalises on students' pre-existing engagement with CVGs and adds a mental health component. This was the initial formulation of Sparky, a CVG with simple mental health content. In this paper we aim to report the process and results of this initial concept testing and implications to help understand whether mental health CVGs such as Sparky are a promising approach to supporting young adolescents' mental health.

\section{Methods}

\section{Recruitment}

High schools were selected from different deciles (a measure of the socio-economic position of a school's student community relative to other schools throughout the country) across the Wellington region. Senior managers or pastoral leaders in seven high schools were approached and all gave consent for students to participate in the study. Student and parental consent was obtained for participants who were involved in the focus groups and workshops.

Presentation and pen and paper responses: In each school, researchers (TF, RP, KS, or SM) gave a presentation to introduce the concept of Sparky. Following each presentation, pen and paper questions designed to elicit preferences and scope initial interest were administered (Table 1). A total of 207 year 9 and year 10 high school students (13-15 years of age; $52 \%$ males; 48\% New Zealand European, 30\% Māori, 12\% Pacific Island) completed the pen and paper question across all groups. Students reported their own demographic characteristics (Table 2). When a student reported more than one ethnic group, they were categorised into Māori, Pacific Island, New Zealand European, or other using the New Zealand Census Ethnicity Prioritisation Method. ${ }^{17}$ We also spoke with students about their perspectives and ideas for

Table 1. Pen and paper response questions.

1) How often do you play apps or casual video games? (Response options: never, about once a week or less, several times a week, or several times a day)

2) What are your thoughts about a casual video game with mental health concepts?

3) If you play apps or casual video games do they make you feel? (Response options: more relaxed, less bored, more focused, more calm, more stressed, more frustrated, more annoyed and other)

4) How old are you?

5) What is your ethnic identity?

6) Have you felt down, depressed or very anxious for more than a few days in a row? (Response options: yes or no) 
Table 2. Demographic characteristics.

\begin{tabular}{|c|c|c|c|c|c|c|c|c|c|c|}
\hline \multirow[b]{2}{*}{ Phase } & \multirow[b]{2}{*}{ Group } & \multirow[b]{2}{*}{$\mathrm{N}$} & \multicolumn{2}{|c|}{ Gender } & \multicolumn{2}{|c|}{ Age in years } & \multicolumn{4}{|c|}{ Ethnicity } \\
\hline & & & Male & Female & 13 & 14 & Māori & $\begin{array}{l}\text { Pacific } \\
\text { Islands }\end{array}$ & $\mathrm{NZE}^{\mathrm{a}}$ & Other \\
\hline \multirow{8}{*}{$\begin{array}{c}\text { Pen and paper } \\
\text { responses }\end{array}$} & 1 & 26 & 10 & 16 & 12 & 14 & 3 & 2 & 12 & 5 \\
\hline & 2 & 28 & 18 & 8 & 12 & 10 & 4 & 4 & 13 & 6 \\
\hline & 3 & 12 & 7 & 5 & 6 & 6 & 7 & & 3 & 2 \\
\hline & 4 & 24 & 14 & 10 & 11 & 13 & 6 & 3 & 14 & 2 \\
\hline & 5 & 43 & 18 & 24 & 17 & 16 & 12 & 2 & 20 & 1 \\
\hline & 6 & 32 & 14 & 15 & 15 & 16 & 5 & 4 & 22 & 1 \\
\hline & 7 & 42 & 22 & 18 & 22 & 18 & 23 & 8 & 10 & 2 \\
\hline & & 207 & 103 & 96 & 95 & 93 & 60 & 23 & 94 & 19 \\
\hline \multirow[t]{5}{*}{ Interviews } & 1 & 15 & 8 & 7 & 10 & 5 & 7 & & 6 & 2 \\
\hline & 2 & 12 & 8 & 4 & 5 & 7 & 2 & & 8 & 2 \\
\hline & 3 & 5 & 5 & & 4 & 1 & 1 & & 4 & \\
\hline & 4 & 10 & 4 & 6 & 3 & 7 & 2 & 3 & 5 & \\
\hline & & 42 & 25 & 17 & 22 & 20 & 12 & 3 & 23 & 4 \\
\hline \multirow[t]{4}{*}{ Workshops } & 1 & 7 & 2 & 5 & 7 & & 3 & & 2 & 2 \\
\hline & 2 & 6 & 4 & 2 & 4 & 2 & 2 & & 4 & 2 \\
\hline & 3 & 8 & 3 & 5 & 2 & 6 & 1 & 1 & 6 & \\
\hline & & 21 & 9 & 12 & 13 & 8 & 6 & 1 & 12 & 4 \\
\hline
\end{tabular}

${ }^{\mathrm{a}}$ New Zealand European.

the game. These discussions were summarised and recorded as field notes.

Focus groups: At the end of each presentation, students were asked if they would be interested in taking part in focus groups to understand their views on existing CVGs and explore their preferences for the new game. From the 207 students involved in the initial consultations, 42 students took part in the focus groups. Focus groups were held at high schools and involved up to 15 participants each. Researchers (TF, $\mathrm{RP}, \mathrm{KS}$ or SM) used a schedule of open-ended questions to guide discussions (Table 3). Each focus group lasted approximately 30 minutes.

Workshops: Once the Sparky prototype was developed, we asked students from the focus groups if they would be interested in providing feedback on the game.
A total of 21 students expressed interest and took part in the workshops. Each workshop comprised 6, 7 or 8 participants. Researchers (TF, RP, KS or SM) used a schedule of open-ended questions (Table 4) to ascertain participants' views of the prototype. Phases of recruitment can be seen in Figure 1.

\section{Data analysis}

We employed a General Inductive Approach (GIA) proposed by Thomas ${ }^{18}$ to identify the broad explicit and implicit themes within the data. First, we closely read the raw data across the three methods. Next, data were clustered into themes, which were highly consistent across pen and paper responses, focus groups and workshops. In the focus groups and workshops, more 
Table 3. Interview questions.

1) If you have played a CVG, when was the last time you played?

2) Can you recall what was happening beforehand?

3) How did you feel when you started?

4) Where were you?

5) How long did you play for?

6) How did you feel doing it?

7) Were there any good things about playing for you?

8) Were there any less good or bad things?

9) What is good and not so good about the prototype?

10) What would improve it?

Table 4. Questions used in workshops.

1) What are your likes and dislikes of the prototype?

2) What are your thoughts on the messages and text?

3) What could be improved?

4) What do you think about the game play?

specific details were recorded about the look and feel of the game. We elected to combine the results; they are structured under three key themes (below), with participant quotes in italics. Although all participants had the opportunity to complete the pen and paper response form, some participants omitted some questions.

\section{Results}

\section{Theme 1: Young adolescents play casual video games and they play for a variety of reasons}

In the pen and paper responses, $91 \%$ (188 out of 207) of young adolescents stated that they play CVGs. Those who reported playing CVGs endorsed a variety of reasons, most commonly to feel more relaxed $(68 \%$; 128 out of 188$)$, more calm $(60 \% ; 112$ out of 188$)$, and less bored $(57 \% ; 107$ out of 188$)$.

Some focus group participants reported playing specific games on their phone to help them feel less stressed or less bored "I have different categories on my phone for different moods....like I will play some games to feel

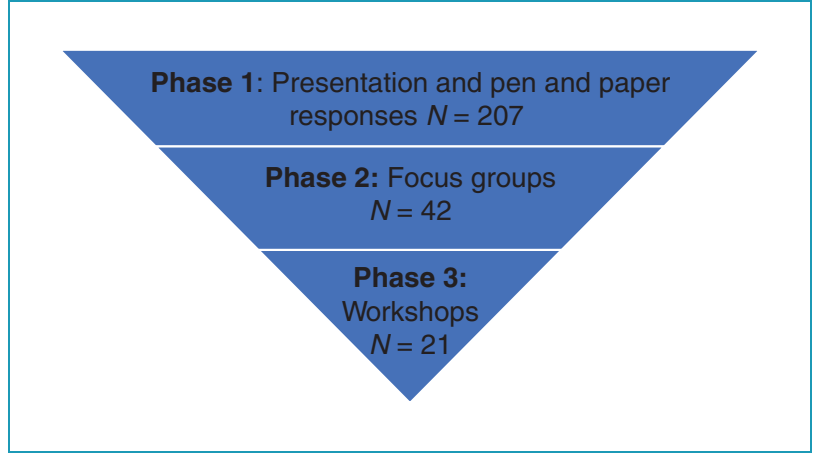

Figure 1. Phases of recruitment.

less stressed or when I am bored". In the workshops, students mentioned that the games they find relaxing often involve simple mechanisms, such as swiping or tapping, and use smooth colours "If you are trying to make it chillaxing then simple stuff is good like a couple of colours", "Games that are relaxing only involve tapping a balloon or swiping some colours so do that I reckon" and "Sometimes I need to do pointless stuff to be less bored like tap a candy and get a reward".

\section{Theme 2: Young adolescents were interested in the idea of a mental health casual video game}

Most participants were interested in playing a mental health CVG. In the pen and paper responses, many thought it was "a cool concept" "beneficial for us students" or "a great idea and could help heaps of people". A number of participants mentioned they thought it was a good idea because CVGs are popular and are designed to be fun. "Nice idea because casual games are fun". "Sounds very similar to games I play so should be fun" and "Seems like the games we play, and I play heaps of them".

Pen and paper responses to the question "have you felt down, depressed or very anxious for more than a few days in a row?' were consistent with the identified need to address mental health concerns. Approximately $66 \%$ (122 out of 186$)$ of respondents who answered this question reported they had felt down, depressed or anxious for more than a few days in a row.

These findings were supported with data from focus group participants, who mentioned that "I think it's a good idea to help heaps of kids who are depressed or anxious cheer up, and I know a lot!", "I feel down sometimes and guess anything to help would [be] good", "Think it's a good idea because you can focus on mental health which is super important, like some friends I know could use this still but at the same time make it enjoyable for teens". 
Consistent with the initial scoping phase, focus group participants identified the need to help young people with their mental health. Although some mentioned that many young people may not wish to talk about their concerns, they thought that a mental health CVG may be a promising and appealing platform for learning skills and supporting conversations about mental health. In the focus groups, participants stated "Good idea because many people like casual games and lots of people don't like talking about their problems", "Good way of incorporating mental wellbeing into a modern theme", "Great. People already spend heaps of time playing games on their phones, so it would be good, important and makes sense to combine both mental health awareness and games" and "Pretty relatable and heaps of people could learn more about mental health".

In general, participants were excited about the idea and interested in helping to develop the game. Some expressed interest in commenting on design aspects of the game, or even, in the case of some workshop participants, contributing graphics "I think the concept is brilliant and am keen to help out", "If it helps with my negative thoughts, let's do it!" "Seems cool as, keen to help I could even make some pictures" and "I've got a cool as image of good and bad thought you could use".

\section{Theme 3: Recommendations: An engaging interface and subtle mental health information}

Participants provided a number of recommendations when asked to write their thoughts about a CVG with mental health concepts. First, in the pen and paper responses, participants mentioned that an engaging interface is important to motivate people to play "when designing the game use clean and easy to look at images and messages" and "you have to make the game simple and fun to play otherwise people will not play and learn about mental health". Many games that students play involve simple, appealing colour palettes. They recommended using "just a few colours", "making it simple with not many colours" and "colours need to flow".

In the focus groups, participants mentioned that it was important to keep the mental health information and skills subtle. They recommended making the game fun as the first priority and advised that mental health concepts should be used discreetly "you have to make it fun otherwise no one will play", "don't be too heavy handed with the messaging and it will work" and "the more subtle with the mental health stuff the better". Workshop participants also thought the game could be used as an adjunct to face-to-face therapy "the game could help you remember stuff you learnt before, kinda like a homework type thing", "you could use the game if you forgot a mental health tip".

\section{Discussion}

In this stepwise exploratory study, we found that many young adolescents reported playing CVGs and endorsed a number of reasons for doing so, such as to relieve stress, feel more relaxed and relieve boredom. In general, young adolescents expressed interest in the idea of a mental health CVG and wanted to take part in the design process. Participants suggested that an engaging interface and a subtle level of mental health information were important features to consider. These preliminary results suggest that a mental health $\mathrm{CVG}$ is a promising approach to build upon.

One key finding from the study was that young adolescents play CVGs and they play them for a number of reasons. It has been well documented that CVGs are popular among adults who play for a variety of reasons. For instance, Stroud ${ }^{19}$ conducted an online survey about the use of CVGs with 10,308 adults ranging from 18 to 80 years. Researchers found CVGs are highly acceptable among adults, especially those aged 60 and older. Researchers also reported that many adults play to relieve stress and enjoy the challenge of CVGs. These results are similar to an online survey by Reinecke $^{20}$ who recruited 1614 adult users of casual games. Reinecke ${ }^{20}$ found that participants who played games, reported an increase in feelings associated with relaxation after playing. The therapeutic potential of this simplistic approach for adolescent however has been less understood despite their high interest in playing CVGs.

Another important finding from the current study was that young adolescents were interested in the idea of a mental health CVG. Many participants stated this was because it is important to help young people with their mental health, especially those who may not wish to talk about their distress. This is an important finding as it builds our preliminary work that suggests young adolescents generally do not actively seek help unless their concerns become serious enough. ${ }^{12}$ Therefore, using an existing and non-threatening model of engagement appears to be a promising opportunity that aligns with young adolescents' current needs and preferences.

Participants stated that using CVGs is a modern way of promoting mental health because CVGs are fun and many young people are familiar with them. Mobile phones and CVGs are embedded into the lives of many adolescents, who typically play 2-3 times per day in short, 5-15-minute bursts. ${ }^{21}$ With such popularity already established, CVGs may provide an attractive platform on which digital mental health developers can capitalize. ${ }^{1420}$ This approach 
can be contrasted with traditional digital mental health approaches, which have attempted to fit therapy onto platforms such as tablets, computers or CD-ROMs. To date, there has been limited research working from a bottom-up approach to match the delivery of mental health content with the way youth are currently using technology. $8,11,22,23$

Using CVGs may have additional advantages, such as providing scalable and non-stigmatizing ways to access therapeutic content for adolescents. Furthermore, young 'digital natives' may prefer this method over psychotherapies with counsellors and therapists, as they are more familiar and comfortable with this platform. ${ }^{24}$ Although the current approach is not designed to treat or elicit disclosures of mental health difficulties, participants reported a high level of acceptability of using this platform to promote mental health content.

Participants gave recommendations such as using simple colours for an engaging interface and a subtle level of mental health information. They reported that it was important for the game to increase in difficulty to ensure it was not too easy or hard. According to flow theory, it is important to design games whereby the skills of the individual are matched with the rules, goals and challenges of the task. ${ }^{25}$ Popular CVGs such as Candy Crush capitalise on this idea by increasing the difficulty of levels as a player moves through the game. These games have also identified the importance of colours. Candy Crush, for example, uses three primary (red, yellow, blue) and three secondary colours (orange, green, purple). This simple palette ensures high contrast to maximise the clarity of images and text on mobile devices. ${ }^{26,27}$ Finally, some students recommended that the game could be used to supplement face-to-face therapy. This idea is important to consider given the importance of reinforcing content discussed in therapeutic contexts. ${ }^{28}$

\section{Strengths and limitations}

This was a small study that used several distinct methodological approaches among seven high schools in the Wellington region of New Zealand. It was an appropriate approach for an exploratory scoping project to help understand the role and popularity of CVGs among young adolescents. Participant responses provided a rationale for developing this idea further and user feedback regarding the design of a mental health CVG. However, this study was not designed to be generalised to other populations hence the small sample size. Future research could attempt to replicate these findings across different populations of adolescents. In addition, it would be interesting to examine or test the mechanisms of change that contribute to the therapeutic effects of CVGs in order to inform the development of Sparky.

\section{Conclusion}

Findings from this stepwise exploratory study suggest that many young adolescents play CVGs and do so for a number of reasons, such as to relieve stress, feel more relaxed and relieve boredom. Participants were interested in the idea of a mental health CVG (Sparky), articulating that it is important to address mental health issues among their cohort. They also noted that CVGs are popular and designed to be fun, therefore this approach offers a modern way of supporting mental health. Students provided design recommendations for the CVG, such as creating an engaging interface and subtle mental health skills and information. Involving adolescents in the design process of a mental health CVG offered an opportunity to increase acceptability and engagement. Results suggest that the concept of a mental health CVG appears to be appealing to young adolescents and should be tested

Acknowledgements: We would like to thank the young people, teachers and schools that were involved with this research.

Contributorship: RP, TF, KS and SM were all involved in the initial scoping phase of Sparky. RP, TF and KS were involved in the focus groups and workshops. RP completed the first draft of the manuscript and completed the data analysis. All authors reviewed and edited the manuscript and approved the final version of the manuscript.

Declaration of conflicting interests: The author(s) declared no potential conflicts of interest with respect to the research, authorship, and/or publication of this article.

Ethical approval: The study was approved by Victoria University of Wellington Human Ethics Committee \#0000026853.

Funding: The author(s) disclosed receipt of the following financial support for the research, authorship, and/or publication of this article: This research was supported by the Digital Futures Activity Funded Project.

\section{Guarantor: RP.}

ORCID iD: Russell Pine (D) https://orcid.org/0000-0002-02243757

Peer review: Lauren Gardner, The University of Sydney, and Kevin O'Brien, East Carolina University, have reviewed this manuscript. 


\section{References}

1. World Health Organization. Depression and other common mental disorders: global health estimates (No. WHO/MSD/MER/2017.2). Geneva: World Health Organization, 2017.

2. Lockett H, Lai J, Tuason C, et al. Primary healthcare utilisation among adults with mood and anxiety disorders: an analysis of the New Zealand health survey. $J$ Prim Health Care 2018; 10: 68-75.

3. Gulliver A, Griffiths K and Christensen H. Perceived barriers and facilitators to mental health help-seeking in young people: a systematic review. BMC Psychiatry 2010; 10: 113.

4. Wilson CJ and Deane FP. Adolescent opinions about reducing help-seeking barriers and increasing appropriate help engagement. J Educ Psychol Consult 2001; 12: 345-364.

5. Hollis C, Falconer CJ, Martin JL, et al. Annual research review: digital health interventions for children and young people with mental health problems-a systematic and Meta-review. J Child Psychol Psychiatry 2017; 58: 474-503.

6. Ebert DD, Zarski AC, Christensen H, et al. Internet and computer-based cognitive behavioral therapy for anxiety and depression in youth: a meta-analysis of randomized controlled outcome trials. PLoS One 2015; 10: e0119895.

7. Fleming TM, Bavin L, Stasiak K, et al. Serious games and gamification for mental health: current status and promising directions. Front Psychiatry 2016; 7: 215.

8. Thabrew H, Fleming TM, Hetrick S, et al. Co-design of eHealth interventions with children and young people. Front Psychiatry 2018; 9: 481.

9. Baumel A, Edan $\mathrm{S}$ and Kane J. Is there a trial bias impacting user engagement with unguided e-mental health interventions? A systematic comparison of published reports and real-world usage of the same programs. Transl Behav Med 2019; 9: 1020-1033.

10. Bowman ND, Schultheiss D and Schumann C. "I'm attached, and I'm a good guy/gal!": how character attachment influences pro-and anti-social motivations to play massively multiplayer online role-playing games. Cyberpsychol Behav Soc Netw 2012; 15: 169-174.

11. Scholten H and Granic I. Use of the principles of design thinking to address limitations of digital mental health interventions for youth. J Med Internet Res 2019; 21: e11528.

12. Fleming T, Dixon R and Merry S. "It"s mean!' the views of young people alienated from mainstream education on depression, help seeking and computerised therapy. $A d v$ Mental Health 2012; 10: 195-203.

13. Fleming T, Stasiak K, Moselen E, et al. Revising computerized therapy for wider appeal among adolescents: youth perspectives on a revised version of SPARX. Front Psychiatry 2019; 10: 802.

14. Limelight Networks. Market research: the state of online gaming - 2019, www.limelight.com/resources/whitepaper/state-of-online-gaming-2019/ (2019, accessed 12 January 2020).

15. Entertainment Software Association. 2019 essential facts about the computer and video game industry, www.th eesa.com/esa-research/2019-essential-facts-about-thecomputer-and-video-game-industry/ (2019, accessed 16 January 2020).

16. Casual games market report 2007, https://issuu.com/casualconnect/docs/casualgamesmarketreport-2007 (accessed 18 November 2019).

17. Poutasi K. Ethnicity data protocols for the health and disability sector. Wellington, New Zealand: Ministry of Health, 2004.

18. Thomas DR. A general inductive approach for analyzing qualitative evaluation data. Am J Eval 2006; 27: 237-246.

19. Stroud MJ and Whitbourne SK. Casual video games as training tools for attentional processes in everyday life. Cyberpsychol Behav Soc Netw 2015; 18: 654-660.

20. . Reinecke L. Games at work: the recreational use of computer games during working hours. Cyberpsychol Behav 2009; 12: 461-465.

21. Brand JE, Jervis J, Huggins PM, et al. 2019. Digital New Zealand 2020. Eveleigh, NSW: IGEA.

22. Mohr DC, Burns MN, Schueller SM, et al. Behavioral intervention technologies: evidence review and recommendations for future research in mental health. Gen Hosp Psychiatry 2013; 35: 332-338.

23. Tuerk PW, Schaeffer CM, McGuire JF, et al. Adapting evidence-based treatments for digital technologies: a critical review of functions, tools, and the use of branded solutions. Curr Psychiatry Rep 2019; 21: 106.

24. Brooks FM, Chester KL, Smeeton NC, et al. Video gaming in adolescence: factors associated with leisure time use. J Youth Stud 2016; 19: 36-54.

25. Csikszentmihalyi M. Flow and the Foundations of Positive Psychology: The Collected Works of Mihaly Csikszentmihalyi. 2014th ed. Netherlands: Springer, 2014, pp. 1-298.

26. Varonis E, Varonis $M$ and Varonis E. Deconstructing candy crush: what instructional design can learn from game design. Int $J$ Info Learning Tech 2015; 32: 150-164.

27. Kultima A. Casual game design values. In: Proceedings of the 13th international MindTrek conference: everyday life in the ubiquitous era. 2009, pp.58-65. New York: ACM.

28. Kazantzis N, Whittington C and Dattilio F. Meta-analysis of homework effects in cognitive and behavioral therapy: a replication and extension. Clin Psychol Sci Pract 2010; 17: 144-156. 